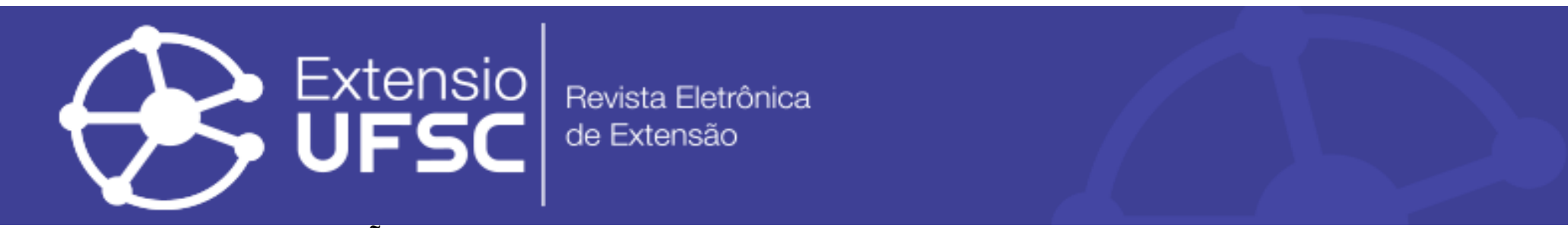

\title{
APLICAÇÃO DE METODOLOGIAS ATIVAS DE ENSINO-APRENDIZAGEM NO PLANEJAMENTO DE ESTRATÉGIAS DE SAÚDE PARA A PESSOA COM \\ DIABETES
}

\author{
Ângelo Bento Silva \\ Universidade do Estado do Amazonas \\ angelobento23@gmail.com \\ Davi Paranhos Fontes Alves \\ Universidade do Estado do Amazonas \\ davifontesalves18@hotmail.com \\ Dayana Souza Ferreira \\ Universidade do Estado do Amazonas \\ dayanasf@outlook.com
}

\author{
Nayara de Oliveira Bitencourt \\ Universidade do Estado do Amazonas \\ nayarabitencourth@hotmail.com \\ Rubenira Mota Rego \\ Universidade do Estado do Amazonas \\ nira_rego@hotmail.com \\ Maurício Bacarin Silva \\ Universidade do Estado do Amazonas \\ mabsilva@uea.edu.br
}

\section{Resumo}

Objetivo: Esse relato de experiência tem a intenção de promover o uso de metodologias ativas de ensino-aprendizagem com uma equipe multiprofissional visando a melhoria do processo de trabalho no cuidado à pessoa com diabetes na Atenção Primária à Saúde (APS), do Sistema Único de Saúde (SUS). Método: A vivência coletiva envolveu as atividades de construção e aplicação de metodologias ativas de ensino-aprendizagem com a equipe de Estratégia Saúde da Família (ESF) e do Núcleo Ampliado de Saúde da Família (NASF), para aprimorar a assistência à pessoa com diabetes. Resultados: Foi possível identificar problemas no processo de trabalho, verificar aquele que a equipe teria governabilidade para intervir e apontar as suas possíveis causas no diagrama de Ishikawa. Conclusão: A Educação Permanente em Saúde Pública amplia o olhar dos profissionais da APS sobre o processo de trabalho ao promover o desenvolvimento de suas capacidades intrínsecas de ação interprofissional da ESF e do NASF. Período: Ocorreu entre maio/2019 a fevereiro/2020. Palavras-chave: Tecnologia Educacional. Equipe de Assistência ao Paciente. Educação em Saúde. Atenção Primária à Saúde.

\section{APPLICATION OF ACTIVE TEACHING-LEARNING METHODOLOGIES IN THE PLANNING OF HEALTH STRATEGIES FOR THE PERSON WITH DIABETES}

Abstract

Objective: This experience report aims to promote the use of active teaching-learning methodologies with a multidisciplinary team with the aim of improving the work process in the care of people with diabetes in Primary Health Care (PHC) of the Unified Health System (SUS). Method: The collective experience involved the activities of construction and application of active teaching-learning methodologies with the Family Health Strategy (FHS) and the Family Health Expanded Support Center (NASF) teams to improve the care for people with diabetes. Results: It was possible to identify problems in the work process, also to verify the problem that the team would have governance to intervene and point out its possible causes in the Ishikawa diagram. conclusion: Permanent Education in Public Health broadens the view of PHC professionals on the work process by promoting the development of their intrinsic capacities of interprofessional action by the FHS and the NASF teams. Period: Occurred between May/2019 to February/2020.

Keywords: Education Technology. Patient Care Team. Health Education. Primary Health Care.

\section{APLICACIÓN DE METODOLOGÍAS ACTIVAS DE ENSEÑANZA-APRENDIZAJE EN LA PLANIFICACIÓN DE ESTRATEGIAS DE SALUD PARA PERSONA CON DIABETES}

Resumen

Objetivo: Este relato de experiencia tiene como objetivo promover el uso de metodologías activas de enseñanzaaprendizaje con un equipo multidisciplinar con el objetivo de mejorar el proceso de trabajo en la atención de personas con diabetes en Atención Primaria de Salud (APS) del Sistema Único de Salud (SUS). Método: La experiencia colectiva involucró las actividades de construcción y aplicación de metodologías activas de enseñanza-aprendizaje con el equipo de salud de la Estrategia Salud de la Familia (ESF) y del Centro Extendido de la Salud de la Familia (NASF) para mejorar la atención a las personas con diabetes. Resultados: Se logró identificar problemas en el proceso de trabajo, verificar en los cuales el equipo tendría capacidad para intervenir y señalar sus posibles causas en el diagrama de Ishikawa. Conclusión: La Educación Permanente en Salud Pública amplía la visión de los profesionales de la APS sobre el proceso de trabajo al promover el desarrollo de sus capacidades intrínsecas de acción interprofesional por parte de la ESF y del NASF. Período: Ocurrió entre mayo/2019 a febrero/2020.

Palabras clave: Tecnología Educacional. Grupo de Atención al Paciente. Educación en Salud. Atención Primaria de Salud. 
Aplicação de metodologias ativas de ensino-aprendizagem no planejamento de estratégias de saúde para a pessoa com diabetes

\section{INTRODUÇÃO}

A vivência no cenário de prática e a imersão no cotidiano de profissionais de saúde remete ao processo constante de construção e desconstrução para o alinhamento do processo de trabalho em uma equipe multiprofissional da Atenção Primária à Saúde (APS), envolvendo a Estratégia Saúde da Família (ESF) e o Núcleo Ampliado de Saúde da Família (NASF). Para que esses processos sejam realizados, adota-se como ferramenta de ensino e aprendizagem a Educação Permanente em Saúde (EPS), pois tem como objetivo promover mudanças na maneira de agir dos profissionais (ALMEIDA et al., 2016). Contudo, implementar a EPS pode configurar como um desafio, pois é necessário levar em consideração variáveis como: tornar o profissional protagonista desse processo, utilizar metodologias que adequem as falhas do processo de trabalho e fornecer autonomia a esse trabalhador (PERES; SILVA; BARBA, 2016).

As metodologias ativas de ensino-aprendizagem (MA) têm se mostrado importantes recursos para uma formação mais completa e complexa dos profissionais da saúde. O foco se desloca do ensino para a aprendizagem e os estudantes são considerados, então, sujeitos de seus próprios processos de construção de saberes. Trata-se de uma abordagem construtivista do conhecimento (CUSTODIO et al., 2013), que é ao mesmo tempo técnica, cognitiva, afetiva e ética. Trata-se, sobretudo, de uma valorização do ser humano que se faz profissional, visando à formação mais ampla daqueles que atuam na área da saúde. Para tratar dos processos que devem dar lugar a essa nova e mais complexa categoria de formação profissional, Anastasiou e Alves (2003) criaram um neologismo, ensinagem, que é um termo usado para significar uma situação de ensino que resulta necessariamente em aprendizagens. Para que haja de fato a ensinagem, uma condição essencial é a parceria entre as pessoas envolvidas no processo de ensino, como, por exemplo, a EPS. Isso significa que se não há aprendizagem, o ensino não se efetivou por completo. Ensinagem remete a um processo em que o professor, o preceptor, o tutor, são considerados mediadores ou aqueles que organizam situações de aprendizagem para que os estudantes, ativamente, aprendam. São, portanto, parceiros da construção pessoal do percurso formativo de cada estudante, atuando não apenas sobre a técnica e a memória (MORGADO, 2009), mas sobre a formação integral da pessoa e do profissional.

Para Morgado (2009), trata-se de um novo modelo de educação, que contribui de forma mais efetiva para o desenvolvimento de posturas profissionais investigativas e críticas e, nesse sentido, para a busca ativa pela solução dos problemas da realidade concreta. Os estudantes são, sob esse ponto de vista, protagonistas de suas aprendizagens e de seu desenvolvimento e 
Aplicação de metodologias ativas de ensino-aprendizagem no planejamento de estratégias de saúde para a pessoa com diabetes

compreendem o significado e a utilidade do que aprendem, sem divorciar os conhecimentos e a vida ou as situações do cotidiano profissional.

Anastasiou e Alves (2003) afirmam que as MA contribuem para o desenvolvimento de operações do pensamento (comparar, resumir, observar, classificar, interpretar, criticar, buscar suposições, imaginar, obter e organizar dados, levantar hipóteses, aplicar fatos e princípios a novas situações, decidir, planejar) à medida que desenvolvem condutas éticas e atitudes necessárias ao conhecimento e ao enfrentamento da realidade. As autoras asseguram que cabe ao professor prover situações em que os grupos possam exercitar o diálogo e a solução coletiva de problemas, desenvolvendo capacidades que somente podem ser mobilizadas pelas atividades em grupos. Nesse sentido, o professor é um facilitador que acompanha, simultaneamente, o desenvolvimento do grupo e está atento aos processos individuais, o que torna as MA estratégias fundamentais de ensinagem nos cenários de prática do SUS.

Cabe destacar que as MA apontam para uma nova concepção a respeito dos significados da formação profissional. Trata-se de agir sobre o desenvolvimento integral e holístico dos profissionais que, para além de detentores de conhecimentos e técnicas, se tornem mais humanos, mais abertos ao diálogo com o outro, mais reflexivos. É importante compreender que estamos nos referindo a um tipo de formação que supera comportamentos de ordem cognitiva, contribuindo para configurar formas de ser pessoais, incidindo sobre a estrutura mais profunda dos indivíduos. Nesse sentido, a formação profissional interfere sobre a forma de ver o mundo, os conhecimentos, o trabalho, a vida. As MA possibilitam o exercício das capacidades e competências requeridas para o trabalho de escuta do outro, de atenção aos pormenores das relações, de trabalho coletivo (RUÉ, 2009).

A ESF trabalha a promoção da saúde em que cabe utilizar as MA para buscar melhores resultados a partir de diversos aspectos na prevenção, tratamento e reabilitação de doenças. Além de buscar o cuidado integral e longitudinal, por meio de atendimentos e acompanhamento contínuo e sistematizado dos usuários com condições crônicas. Contudo, a maior parte das ações realizadas pelas equipes de saúde ainda se concentra no desenvolvimento de consultas, principalmente de enfermagem e de medicina (SASS et al., 2012).

As Doenças Crônicas Não Transmissíveis (DCNT) representam uma ameaça para a saúde e desenvolvimento a todas as nações. As DCNT se constituem como o grupo de doenças de maior magnitude no país, atingindo, especialmente, as populações mais vulneráveis. O diabetes é um exemplo e está associado a comorbidades que comprometem a qualidade de vida do paciente além de ser um grave problema para a saúde pública (SOUSA et al., 2018). A maioria das comorbidades é consequência de uma assistência que não considera as especificidades do 
Aplicação de metodologias ativas de ensino-aprendizagem no planejamento de estratégias de saúde para a pessoa com diabetes

cuidado das cronicidades relacionadas com o diabetes e utiliza modelos assistenciais inadequados (SALCI; MEIRELLES; SILVA, 2018).

O desafio para os serviços de saúde é garantir o acompanhamento sistemático dos portadores de diabetes, evitar a evolução de agravos da doença e desenvolver ações de prevenção e promoção à saúde (CARVALHO-FILHA; NOGUEIRA; MEDINA, 2014). A APS possui um papel primordial no cuidado com as DCNT, dentre elas o diabetes, pois na APS é feito o acompanhamento integral e multiprofissional desses pacientes, que vai desde o diagnóstico ao tratamento (RADIGONDA et al., 2016, p. 116).

A APS tem como característica a descentralização, a capilaridade e maior proximidade com as pessoas. Deve ser o contato preferencial dos usuários, a principal porta de entrada e o centro de comunicação com toda a Rede de Atenção à Saúde (RAS). Por isso, é fundamental que ela se oriente pelos princípios da universalidade, da acessibilidade, do vínculo, da continuidade do cuidado, da integralidade da atenção, da responsabilização, da humanização, da equidade e da participação social (BRASIL, 2017).

As transformações das práticas de saúde são construídas a partir da problematização desse processo de trabalho e da realidade do território, além de uma reflexão crítica de modo a gerar um processo de ensino-aprendizagem. A EPS no contexto da ESF e do NASF é essencial para que haja uma melhor concepção da gestão do cuidado em saúde e o empoderamento de instrumentos adequados para intervir nas necessidades de saúde dos indivíduos e, assim, resultar em um bom desenvolvimento do trabalho em equipe (ALMEIDA et al., 2016, PINHEIRO; AZAMBUJA; BONAMIGO, 2018). As MA constituem uma estratégia baseada na problematização, na construção do conhecimento a partir de experiências reais e, assim, no desenvolvimento de habilidades para lidar com os desafios cotidianos, por isso são tão importantes no processo da EPS (COLARES; OLIVEIRA, 2018).

Outras ferramentas de mudança organizacional utilizadas pela gestão enriquecem a aplicação de MA, podendo ser consideradas ideias inovadoras no cenário da APS, sendo que foram criadas para o planejamento estratégico no setor industrial. O Diagrama de Ishikawa ou de Causa e Efeito demonstra a relação entre o problema e as possíveis causas pensadas pelos trabalhadores, visa ajudar o trabalho em equipe, melhora habilidades de raciocínio lógico e amplia o conhecimento profissional, obtendo resultados mais eficazes para colaboração de todas pessoas envolvidas. Foi desenvolvido por Kaoru Ishikawa, em 1943, e desde então é também amplamente difundido em setores de gestão de qualidade da área da saúde (FIORIN et al., 2016, ZANFERRARI et al., 2016, LIMA et al., 2019, SILVA et al., 2019). 
Aplicação de metodologias ativas de ensino-aprendizagem no planejamento de estratégias de saúde para a pessoa com diabetes

Visto o exposto, esse artigo tem como objetivo relatar a experiência dos discentes do curso de Especialização em Saúde Pública com ênfase em Estratégia Saúde da Família, da Escola de Saúde Pública da Secretaria Municipal de Saúde da Prefeitura de Manaus (ESAP/SEMSA/Manaus/AM), como também apresenta as atividades extensionistas do acordo de cooperação técnica nº 006/2019, na forma prevista na resolução CNE/CES nº 1/2007 para cursos em Saúde Pública, por intermédio da Universidade do Estado do Amazonas (UEA). Assim, caracterizando a integração ensino-serviço, as atividades de extensão universitária vieram oportunizar a construção e aplicabilidade de um projeto de intervenção desenvolvido pelos profissionais pós-graduandos inseridos no cenário de prática da APS, com a orientação de um docente da UEA. O projeto de intervenção tinha como propósito fortalecer a EPS com a equipe da ESF e do NASF, visando a melhoria do processo de trabalho e, consequentemente, do cuidado prestado a pessoa com diabetes, além de fomentar as discussões sobre a temática. A vivência desse momento de diálogo entre os pares de uma equipe multiprofissional compartilha como foi experimentar essa realidade e os desafios enfrentados para a aplicação de MA no cenário de prática do SUS.

\section{MATERIAIS E MÉTODOS}

Trata-se de um relato de experiência sobre um projeto de intervenção desenvolvido por cinco discentes do curso de Especialização em Saúde Pública, com ênfase em Estratégia Saúde da Família, da ESAP/SEMSA/Manaus/AM com a cooperação técnica e coordenação pedagógica da UEA. Esse curso tem como objetivo inserir os discentes nos serviços ofertados pela SEMSA/Manaus/AM, especificamente na APS, na modalidade de pós-graduando bolsistas, estando alinhado o processo de ensino e aprendizagem à prática da APS. As atividades desenvolvidas estão aprovadas pelo Comitê de Ética da Universidade Federal do Amazonas (CAAE 24092919.5.0000.5020).

O processo de construção do projeto de intervenção utilizou MA e seu desenvolvimento ocorreu entre os meses de maio de 2019 a fevereiro de 2020, por discentes imersos na equipe multiprofissional de saúde de uma Unidade de Saúde da Família do município de Manaus/AM.

Para construção do projeto e elaboração de estratégias planejadas e alinhadas com a equipe foram utilizadas algumas ferramentas de planejamento. Primeiramente, os problemas do território foram elencados e um deles foi selecionado para intervenção por meio de matrizes, como a matriz de priorização de problemas, a matriz de decisória e de identificação e a matriz de relevância de atores sociais, que utilizam quadros como recurso visual para viabilizar o consenso 
Aplicação de metodologias ativas de ensino-aprendizagem no planejamento de estratégias de saúde para a pessoa com diabetes

da tomada de decisão pela equipe. A reflexão em equipe sobre os atores sociais envolvidos que seriam relevantes no planejamento e execução do projeto elencou membros da comunidade, da equipe de saúde, da gestão local, distrital e municipal. O diálogo entre todos os membros permitiu a aproximação de metas para o processo de trabalho, partindo de uma visão micro para macro, o pensamento estratégico compartilhado corrobora na construção das etapas de intervenção sobre o problema apresentado.

Sobre este contexto, a equipe de intervenção foi acompanhada por um docente da UEA e foi composta pelos discentes imersos no serviço, que vivenciaram o delineamento de uma árvore explicativa de problemas para refletir sobre as causas e as consequências do problema e, assim, elaborar os seus descritores e eleger o nó crítico que é considerado a causa passível de sofrer intervenção. Após a aplicação das ferramentas acima citadas, a equipe pactuou que o projeto de intervenção ocorreria sobre o problema eliciado.

A execução do referido projeto de intervenção foi definida para ser realizada nas seguintes etapas: 1- Motivação da equipe da APS envolvida com encontro para um estudo de caso; 2 Levantamento das falhas do processo de trabalho pela própria equipe com tempestade de ideias; 3- Identificação das causas do problema pela construção da equipe do diagrama de causa e efeito de Ishikawa.

A motivação buscou a aproximação dos membros da equipe para aumentar a empatia entre todos envolvidos, que ocorreu no primeiro encontro. $\mathrm{O}$ estudo de caso foi selecionado pela equipe de intervenção para fomentar na equipe reflexões acerca do problema eliciado entre os discentes, por meio de uma situação-problema vivenciado por um usuário do estabelecimento de saúde em que a equipe multiprofissional estava inserida e foi apresentado no segundo encontro. A partir dessas reflexões, o facilitador deu a oportunidade de todos contribuírem com a tempestade de ideias para a equipe decidir qual era o problema a sofrer a intervenção.

O diagrama de Ishikawa foi aplicado no terceiro encontro com o objetivo de a equipe identificar as causas reais do problema em pauta de acordo com as categorias propostas, que são: método, fatores relacionados com o procedimento; material, relacionado com os componentes, insumos; mão de obra, relacionado com a falha humana, pessoas; máquina, relacionado com equipamentos; medida, relacionado com controle do processo, monitoramento; meio ambiente, relacionado com infraestrutura.

O grupo foi composto por dez profissionais da equipe de saúde, sendo 4 homens e 6 mulheres com idade entre 23 e 56 anos, de várias categorias, entre eles: dois enfermeiros, um profissional de educação física, um fisioterapeuta, um farmacêutico, um assistente social, um agente comunitário de saúde, um psicólogo, um médico e um técnico de enfermagem. 
Aplicação de metodologias ativas de ensino-aprendizagem no planejamento de estratégias de saúde para a pessoa com diabetes

As etapas da intervenção foram executadas com duração de duas horas cada e lançaram mão de uma abordagem centrada nos sujeitos envolvidos no processo, sendo o facilitador um dos discentes, que também fazia parte da equipe de saúde que sofria a intervenção. Para atingir os objetivos traçados foram utilizadas dinâmicas de grupo, sempre no início das atividades, de modo a promover integração e melhorar o ambiente, antes de iniciar a roda de conversa e os movimentos planejados com a equipe para executar o plano de intervenção.

\section{RESULTADOS E ANÁLISES}

O cotidiano e a imersão no trabalho da equipe multiprofissional da ESF e do NASF pelos cinco discentes possibilitaram notar falhas no processo de trabalho no cenário de prática da APS. A falha relacionada com o manejo e o cuidado à pessoa com diabetes pode ocasionar complicações que afetam principalmente o sistema cardiovascular e o sistema nervoso, além de poder ocasionar hospitalizações (FONTBONNE et al., 2018). A ideia de sensibilizar a equipe de profissionais envolvidos no cuidado à pessoa com diabetes para melhorar a qualidade da assistência prestada culminou na construção do projeto de intervenção.

As MA utilizadas como estratégia de fortalecimento da EPS apresentam elementos que facilitam o processo educativo e permitem participação ativa dos sujeitos envolvidos (PINHEIRO; AZAMBUJA; BONAMIGO, 2018). A metodologia da roda de conversa utilizada despertou na equipe sentimentos próprios, estimulou o pensamento crítico-reflexivo e a autoestima ao compartilhar suas experiências, sendo sujeitos ativos no processo de aquisição e transmissão de conhecimento. Tal estratégia de ensino-aprendizagem busca a construção coletiva tanto do levantamento de problemas quanto das causas para solucioná-los e, dessa forma, desenvolver novos conhecimentos, habilidades e melhorar o relacionamento interpessoal com a equipe de trabalho (COLARES; OLIVEIRA, 2018).

O primeiro encontro teve o intuito de promover integração e minimizar a resistência da equipe quanto ao projeto. A vivência da dinâmica de grupo do "falar pelas costas" foi realizada a fim de promover maior integração da equipe e trabalhou a socialização entre os membros da equipe presente, despertando nos profissionais o desejo de usar seus talentos para se ajudarem mutuamente. O entrosamento da equipe é importante para que o levantamento dos problemas seja percebido por eles e dessa forma a intervenção se torne mais interessante e seja possível alcançar a participação de todos da equipe (GODOI; LEITE,2020).

No segundo encontro um estudo de caso foi apresentado com uma situação problema, para trazer à tona as impressões da equipe de saúde sobre a assistência à pessoa com diabetes. Por 
Aplicação de metodologias ativas de ensino-aprendizagem no planejamento de estratégias de saúde para a pessoa com diabetes

meio da tempestade de ideias, todos os participantes foram estimulados a participar do diálogo e contribuir à medida que as ideias emergiam. A equipe trabalhou na identificação dos problemas e no consenso de propostas para a resolução dos mesmos. Nesta etapa foi necessário administrar as diferenças, sem rejeitar ou censurar nenhuma ideia, mas buscando o enfoque no tema principal.

Essa metodologia promove o pensamento reflexivo sobre realidade, abordando os diferentes olhares que são compartilhados com a equipe por cada trabalhador de saúde. Eles são os protagonistas do processo de ampliar os horizontes na reflexão das situações problema e o mediador é um facilitador que estimula o consenso do grupo na tomada de decisão coletiva. É característico da metodologia que os atores envolvidos ouçam, perguntem, reflitam e, a partir disso, a equipe construa conhecimento (COLARES; OLIVEIRA, 2018).

Ao analisar o construto obtido ao término da segunda etapa, obteve-se como resultado o seguinte problema: "a fragilidade da equipe multiprofissional no atendimento às demandas relacionadas à diabetes". Para chegar a esse resultado, optou-se pelo consenso entre todos os profissionais envolvidos durante a realização do projeto de intervenção, por meio do diálogo para encontrar um problema que pudesse sofrer intervenção e fosse utilizado para a construção do diagrama de Ishikawa.

A equipe de saúde identificou apenas quatro das categorias do diagrama de Ishikawa para apresentar causas para o problema principal, que foi refletido pela equipe e está representado na extremidade direita do eixo horizontal central. Os eixos diagonais identificam as categorias em que a equipe trouxe contribuições sobre as possíveis causas do problema (Figura 1). Por meio da metodologia adotada, foi possível organizar, resumir e descobrir conhecimentos sobre possíveis causas para determinado efeito, diante disso, tornou-se viável aperfeiçoar o processo, registrar as causas potenciais para os problemas e envolver toda equipe multiprofissional.

Em estudo semelhante realizado para levantar as principais falhas relacionadas com a assistência odontológica de idosos, essa ferramenta foi importante para compreender as múltiplas causas para o problema levantado na pesquisa (ARAÚJO et al., 2016).

Diante do que foi identificado para cada eixo, é possível observar que somente sobre o eixo método seria possível haver uma intervenção, pois seria o único que haveria a possibilidade de gerenciamento de ação pela equipe de saúde. Dentre as causas destacaram-se a falta de atividade de educação em saúde para a pessoa com diabetes sobre a importância das consultas, sobre o uso dos medicamentos e do glicosímetro, a falha na realização do monitoramento e cadastro de pessoas com diabetes e a falta de comunicação entre os profissionais.

Uma pesquisa realizada na APS no município de Caxias, no Maranhão, corrobora com esses achados, pois demonstrou que não existem atividades de educação em saúde na 
Aplicação de metodologias ativas de ensino-aprendizagem no planejamento de estratégias de saúde para a pessoa com diabetes

comunidade e não há distribuição de materiais educativos. Além disso, verificou-se que apesar dos profissionais de saúde realizarem atividades de promoção à saúde para hipertensos e/ou diabéticos, não há planejamento dessas ações, nem existe uma continuidade na oferta das mesmas (CARVALHO-FILHA; NOGUEIRA; MEDINA, 2014).

Pesquisa semelhante realizada no município de Divinópolis, em Minas Gerais, identificou que o fluxo de atendimento das pessoas com diabetes do município não é bem definido, além de problemas para realização do cadastro desses usuários, para realização de acompanhamento regular e adequado nas unidades de saúde e marcação de consultas (GAMA; GUIMARÃES; ROCHA, 2017).

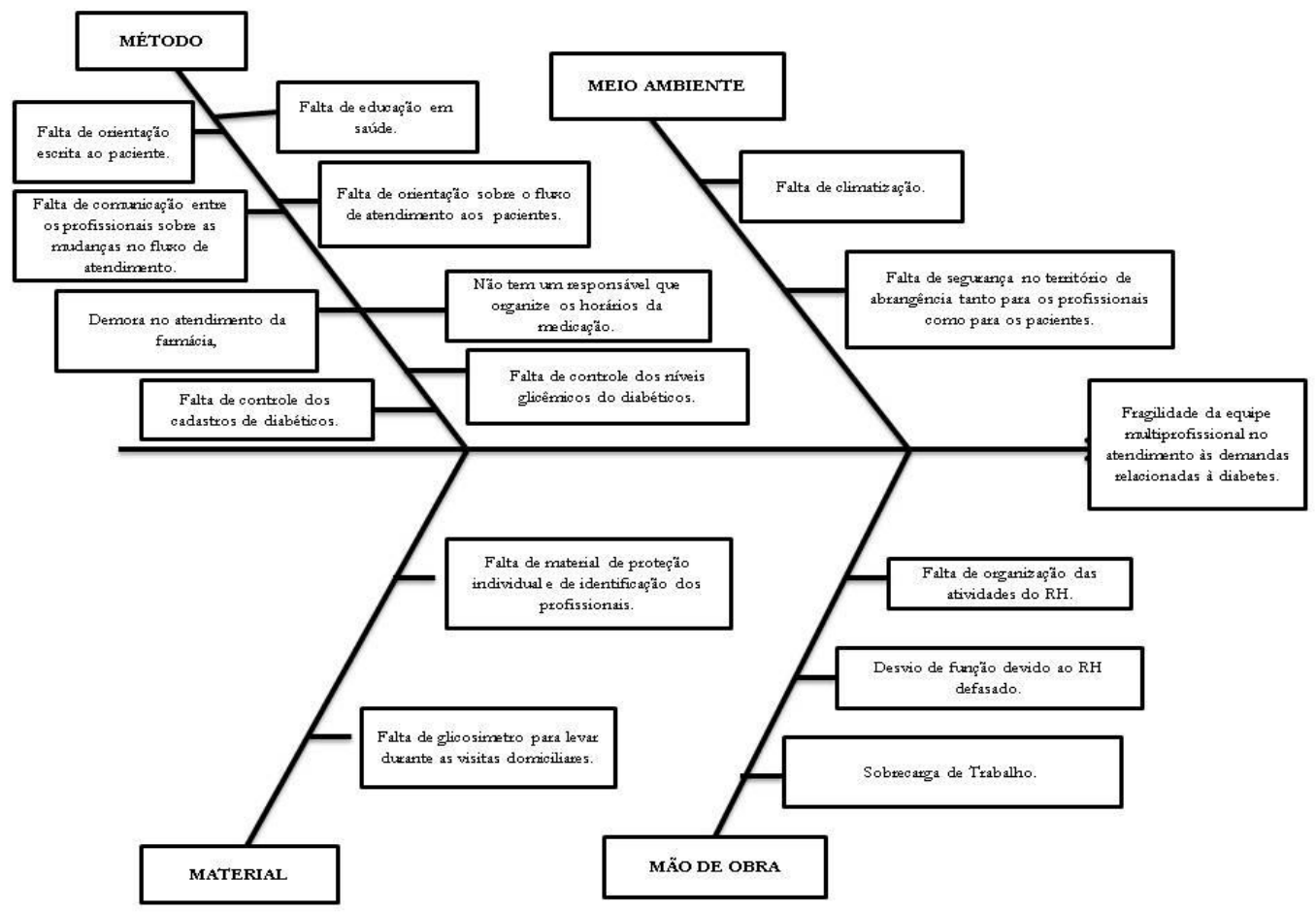

Figura 1: - Diagrama de Ishikawa construído pela equipe multiprofissional de saúde na APS. O problema principal refletido pela equipe está representado na extremidade direita do eixo horizontal central. Os eixos diagonais identificam as categorias em que a equipe trouxe contribuições sobre as possíveis causas do problema. Fonte: elaborada pelos autores.

O próximo encontro seria para o planejamento das ações e pactuação do papel de cada profissional nesse processo, com base nas causas identificadas no diagrama de Ishikawa. Contudo, com a interrupção do projeto devido à pandemia do novo coronavírus, não foi possível dar continuidade na intervenção planejada e não houve uma mudança significativa do processo de trabalho da equipe de saúde e nem no cuidado à pessoa com diabetes. No decorrer do processo, apresentaram-se algumas outras dificuldades, a exemplo da troca do profissional médico da ESF e da baixa participação principalmente dos agentes comunitários de saúde. 
Aplicação de metodologias ativas de ensino-aprendizagem no planejamento de estratégias de saúde para a pessoa com diabetes

Ainda que o estudo tenha apresentado algumas limitações, a vivência junto à equipe propiciou encontros participativos e dialogados, com fortalecimento de vínculos entre os diferentes atores envolvidos no processo. No último encontro foi possível observar mais fluidez e envolvimento nas discussões, muito provavelmente pela familiaridade conquistada com a temática e com as ferramentas utilizadas, baseado na construção compartilhada, o que permitiu maior troca de experiências.

\section{CONSIDERAÇÕES FINAIS}

O presente artigo teve como o seu principal objetivo relatar a experiência durante a elaboração e implementação de um projeto de intervenção sobre as falhas identificadas no processo de trabalho de uma equipe multiprofissional durante a assistência à pessoa com diabetes. Diante do exposto, foi possível apreciar o movimento da equipe sobre uma falha por ela identificada, assim como a reflexão das causas que levam ao aparecimento dessa falha.

O desenvolvimento do projeto, mesmo que não finalizado em decorrência da pandemia do novo coronavírus, permitiu verificar a importância da aplicação de MA para a realização da EPS no processo de trabalho multiprofissional, que contribui para a comunicação entre todos membros da equipe da ESF e do NASF, assim como a necessidade de identificar e problematizar os desafios enfrentados na realidade do território e na oferta dos serviços de saúde.

O levantamento das causas de falhas identificadas possibilita o desenvolvimento de outros projetos para a discussão sobre o cotidiano do trabalho. A reflexão facilita a intervenção e a ressignificação do processo de trabalho pelos atores envolvidos ao apresentar as potências e fortalezas da equipe multiprofissional de saúde da APS.

\section{REFERÊNCIAS}

ALMEIDA, J. R. S.; BIZERRIL, D. O.; SALDANHA, K. de G. H.; ALMEIDA, M. E. L. Educação Permanente em Saúde: uma estratégia para refletir sobre o processo de trabalho. Revista da ABENO, v.16, n.2, p.7-15, 2016. Disponível em: https://revabeno.emnuvens.com.br/revabeno/article/view/248.DOI: https://doi.org/10.30979 /rev.abeno.v16i2.248. Acesso em: 20 de ago. 2020.

ANASTASIOU, Lea das Graças Camargos; ALVES, Leonir Pessate. (orgs.). Processos de ensinagem na universidade: pressupostos para as estratégias de trabalho em aula. Joinville, SC: UNIVILLE, 2003. 
Aplicação de metodologias ativas de ensino-aprendizagem no planejamento de estratégias de saúde para a pessoa com diabetes

ARAÚJO, I. D. T. et al. Atenção à Saúde Bucal de Idosos Institucionalizados na ótica da Gestão e da Atenção Primária. In: Congresso Nacional de Envelhecimento Humano, 2016, Natal. Anais. Campina Grande: Editora Realize, 2016. Disponível em: https://www.editorarealize.com.br/editora/anais/cneh/2016/TRABA LHO_EV054_MD2_SA10_ID345_15082016145750.pdf. Acesso em: 20 de ago. 2020.

BRASIL. Ministério da Saúde. Portaria GM/MS no 2.436, de 21 de setembro de 2017. Aprova a Política Nacional de Atenção Básica, estabelecendo a revisão de diretrizes para a organização da Atenção Básica, no âmbito do Sistema Único de Saúde (SUS). Brasília: Diário Oficial da União, 2017.

CARVALHO-FILHA, F. S. S.; NOGUEIRA, L. T.; MEDINA, M. G. Avaliação do controle de hipertensão e diabetes na Atenção Básica: perspectiva de profissionais e usuários. Saúde Debate [online], Rio de Janeiro, v.38, n.spe, p. 265-278, out. 2014. Disponível em: https://www.scielo.br/j/sdeb/a/cz7Qw4r6VFqGk5cXT9G5J5j/?lang=pt. DO: https://doi.org/10.5935/0103-1104.2014S020. Acesso em: 31 de ago. 2020.

COLARES, K. T. P.; OLIVEIRA, W. Metodologias Ativas na formação profissional em saúde: uma revisão. Revista Sustinere, Rio de Janeiro, v.6, n.2, p.300-320, jul-dez. 2018. Disponível em: https://www.e-publicacoes.uerj.br/index.php/sustinere/article/view/36910. Acesso em: 09 set. 2020.

CUSTODIA, J. F. et al. Práticas didáticas construtivistas: critérios de análise e caracterização. TED, Bogota, n. 33, p.11-35, 2013.

FIORIN, J. M. A. et al. Uso do diagrama de ishikawa associado ao planejamento estratégico: experiência na graduação em enfermagem. Revista UNINGÁ Review, [S.1.], v. 26, n. 3, jun. 2016. Disponível em: http://revista.uninga.br/index.php/uningareviews/article/view/1809. Acesso em: 27 jul. 2021.

FONTBONNE, A. et al. Relações entre os atributos de qualidade de atenção aos usuários hipertensos e diabéticos na Estratégia Saúde da Família e o controle dos fatores prognósticos de complicações. Cadernos Saúde Coletiva [online], Rio de Janeiro, v.26, n.4, p.418-424, nov. 2018. Disponível em: https://www.scielo.br/j/cadsc/a/gbXRg6ZChTNRxktVPDFTzGC/?lang=pt. DOI: https://doi.org/10.1590/1414-462X201800040208. Acesso em: 01 de set. 2020.

GAMA, C. A. P.; GUIMARÃES, D. A.; ROCHA, G. N. G. Diabetes Mellitus e atenção primária: percepção dos profissionais sobre os problemas relacionados ao cuidado oferecido às pessoas com diabetes. Pesquisas e Práticas Psicossociais, São João del Rei, v.12, n.3, p.1-16, set-dez. 2017. Disponível em: http://pepsic.bvsalud.org/scielo.php?script=sci_arttext\&pid=S180989082017000300013. ISSN 1809-8908. Acesso em: 31 de ago. 2020.

GODOI, B. B.; LEITE, L. F. A. Educação permanente em agentes comunitários de saúde: experiência de um projeto de intervenção. Extensio: R. Eletr. de Extensão. Florianópolis, v.17, n.35, p.138-46. 2020. Disponível em: https://periodicos.ufsc.br/index.php/extensio/article/view/1807-0221.2020v17n35p138/43033. DOI: https://doi.org/10.5007/1807-0221.2020v17n35p138. Acesso em: 01 de set. 2020.

LIMA, N. S. et al. Ishikawa Diagram: Causes and Solutions of Hiv Infection in Nursing Professionals Due to Sharps Use. Revista de Pesquisa: Cuidado é Fundamental Online, v. 11, n. 3, p. 707, 2019. 
Aplicação de metodologias ativas de ensino-aprendizagem no planejamento de estratégias de saúde para a pessoa com diabetes

MORGADO, José Carlos. Processo de Bolonha e Ensino Superior num mundo globalizado. Educ. Soc., Campinas, vol. 30, n. 106, p. 37-62, jan./abr. 2009.

PERES, C.; SILVA, R. F.; BARBA, P. C. S. D. Desafios e potencialidades do processo de educação permanente em saúde. Trab. Educ. Saúde [online], Rio de Janeiro, v.14, n.3, p.783-801, set. 2016. Disponível em: www.scielo.br/scielo.php?script=sci_arttext\&pid=S198177462016000300783. DOI: https://doi.org/10.1590/1981-7746-sol00016. Acesso em: 01 de set. 2020

PINHEIRO, G. E. W.; AZAMBUJA, M. S.; BONAMIGO, A. W. Facilidades e dificuldades vivenciadas na Educação Permanente em Saúde, na Estratégia Saúde da Família. Saúde Debate, Rio de Janeiro, v.42, n.especial 4, p.187-197, dez. 2018. Disponível em: https://www.scielo.br/scielo.php?script=sci_arttext\&pid=S0103-11042018000800187. DOI: https://doi.org/10.1590/0103-11042018S415. Acesso em: 05 de set. 2020.

RADIGONDA, B. et al. Avaliação do acompanhamento de pacientes adultos com hipertensão arterial e ou diabetes melito pela Estratégia Saúde da Família e identificação de fatores associados, Cambé-PR, Brasil, 2012. Epidemiol. Serv. Saúde [online], Brasília, v.25, n.1, p.115-126, jan-mar. $2016 . \quad$ Disponível em:

https://www.scielo.br/j/ress/a/c8V4Z7TFVSHHkdCXhch97by/abstract/?lang=pt. $\quad$ DOI: https://doi.org/10.5123/S1679-49742016000100012. Acesso em: 05 de set. 2020.

RUÉ, Joan. El aprendizaje autónomo en educación superior. Madrid: Narcea, 2009.

SASS, A. et al. Depressão em idosos inscritos no Programa de Controle de hipertensão arterial e diabetes mellitus. Acta Paul. Enferm. [online], São Paulo, v.25, n.1, p.80-85. 2012. Disponível em: https://www.scielo.br/j/ape/a/vnQtn7dfmFJMmXyfqgmpTSn/abstract/?lang=pt. $\quad$ DOI: https://doi.org/10.1590/S0103-21002012000100014. Acesso em: 05 de set. 2020.

SILVA, D. F. et al. Causas e Estratégias de Soluções para Hanseníase em Crianças: Diagrama de Ishikawa. Revista de Pesquisa: Cuidado é Fundamental [Online], v. 11, n. 3, p. 739, 2019.

ZANFERRARI, P. M. et al. Desenvolvimento e aplicação de instrumento administrativo para orientação das pesquisas em telefonoaudiologia. Revista CEFAC, v. 18, n. 2, p. 459-469, 2016.

Recebido em: 27/09/2020

Aceito em: 13/08/2021 